\title{
Collectif, Les récits historiques entre Orient et Occident $\left(\mathrm{XI}-\mathrm{XV} \mathrm{V}^{e}\right.$ siècle)
}

\section{Pierre Courroux}

\section{OpenEdition}

\section{Journals}

Édition électronique

URL : https://journals.openedition.org/ccm/8142

DOI : $10.4000 / \mathrm{ccm} .8142$

ISSN : 2119-1026

\section{Éditeur}

Centre d'études supérieures de civilisation médiévale/Université de Poitiers

\section{Édition imprimée}

Date de publication : 1 septembre 2021

Pagination : 284-286

ISBN : 978-2-490783-10-6

ISSN : 0007-9731

Référence électronique

Pierre Courroux, "Collectif, Les récits historiques entre Orient et Occident ( $x l^{e}-x v^{e}$ siècle) 》, Cahiers de civilisation médiévale [En ligne], 255 | 2021, mis en ligne le 01 septembre 2021, consulté le 02 février 2022. URL : http://journals.openedition.org/ccm/8142 ; DOI : https://doi.org/10.4000/ccm.8142

\section{cc)}

La revue Cahiers de civilisation médiévale est mise à disposition selon les termes de la Licence Creative Commons Attribution - Pas d'Utilisation Commerciale - Pas de Modification 4.0 International. 
Les récits historiques entre Orient et Occident (XI $I^{e}-X V^{e}$ siècle), Irene Bueno et Camille RouXPETEL (dir.), Rome, École française de Rome (Collection de l’École française de Rome, 554), 2019.

Les directrices de l'ouvrage se revendiquent, dans leur introduction d'une approche renouvelée de l'historiographie, à la suite de Bernard Guenée, mais aussi de Gabrielle Spiegel. Ils reprennent à Étienne Anheim et Pierre Chastang la formule de l'écriture historique comme un « fait social total » (p. 11). Cependant, dès les premières pages, elles laissent volontairement de côté tout un pan des études actuelles sur les chroniques, à savoir l'étude des mécanismes et procédés qui président à la mise à l'écrit. Comme elles le reconnaissent : « C'est donc moins l'écriture de l'histoire en tant que telle que comme observatoire privilégié des circulations, des points de contact, des relations entre la Méditerranée orientale et occidentale, entre des chrétientés d'Orient et d'Occident, qui nous retiendra au fil de ces pages. » (p. 3) Cette vision clairement exposée préside en effet à la première partie de l'ouvrage, qui en forme environ la moitié, mais on verra que dès la seconde partie, plusieurs articles sont en fait des études de cas sur les procédés d'écriture utilisés par certains historiens et délaissent parfois totalement ou presque la question du transfert des savoirs entre Orient et Occident (Marco Bais et Sergio La Porta).

La préface de Frédéric Gabriel (p. 13-19) revient sur l'importance du modèle biblique pour la production de récits historiques. Il remarque qu'écrire l'histoire pour prouver des arguments théologiques ou religieux ne fait que continuer le modèle même du Judaïsme et du christianisme, qui racontent l'histoire pour prouver leur vérité. Cette préface donne le ton pour un recueil d'études qui placent au cœur de leurs interrogations l'histoire religieuse et l'ecclésiologie. Les articles sont ensuite répartis autour de trois axes : le premier concerne l'histoire comme témoin et vecteur de transmission des savoirs, mais aussi de l'idéologie. Il s'agit donc d'une approche très classique de l'historiographie médiévale, qui se révèle toutefois fertile à travers l'originalité des œuvres et des sujets abordés. L'accent est mis sur le rôle de la cour des Papes comme lieu de transmission des savoirs, carrefour où arrivent des récits et d'où ils se répandent dans d'incessants mouvements d'aller-retour entre Orient et Occident.

Dans le premier article (p. 23-49), Benjamin Weber, présente l'historiographie de l'invasion au $\mathrm{VI}^{\mathrm{e}} \mathrm{s}$.
d'Himyar, royaume juif d'Arabie du sud, par Kaleb, roi éthiopien. Il compare les mémoires orientales de l'événement (byzantines, éthiopiennes, syriaques, islamiques) et le récit occidental à la cour des Papes $\mathrm{du} \mathrm{Xv}^{\mathrm{e}} \mathrm{s}$. Il part d'ailleurs d'un récit italien tardif pour tenter de reconstruire la chaîne de transmission mémorielle de l'événement : pourquoi les ambassadeurs éthiopiens ont-ils conté cette histoire vieille de neuf siècles à Flavio Biondo? À travers un savant survol de nombreux siècles, régions et langues, l'a. retrace une transmission indirecte et chaotique, où le texte-clé est le Martyre d'Aréthas, texte grec traduit en arabe qui circula dans toute la méditerranée orientale. Le succès et la transmission du récit sont dus à la fois à un investissement idéologique par les souverains d'Éthiopie à la fin du Moyen Âge, mais aussi au fait que le texte correspondait aux attentes des Papes dans leur politique d'alliance contre les musulmans. Cette excellente étude correspond pleinement à l'approche présentée dans l'introduction de l'ouvrage, mais est aussi un bel exemple de méthodologie historique, même si l'a. reconnaît volontiers (p. 35) qu'elle mériterait d'être étendue à plus de sources arabes, souvent inaccessibles et non traduites, pour être exhaustive.

Un second article d'Irene Bueno (p. 51-71) s'intéresse à l'histoire arménienne telle qu'elle fut rédigée en Occident au XIV ${ }^{\mathrm{e}}$ s., à travers deux œuvres destinées notamment à la cour pontificale. Daniel de Tabriz, dont l'œuvre resta confidentielle, écrivit à Avignon un traité défendant l'orthodoxie de l'église arménienne face aux accusations qui pouvaient avoir cours en Occident. Héthoum de Korykos, qui connut un certain succès, rédigea lors d'un séjour à Poitiers une histoire des Mongols visant à promouvoir une alliance entre les Arméniens et l'Occident. Tous insistent sur le nécessaire secours à apporter aux Arméniens de Cilicie pour défendre la foi chrétienne.

Partant du constat que Christophe Colomb partit à la recherche du Grand Khan en 1492, Thomas Tanase (« La figure du grand-khan à travers la littérature occidentale de Marco Polo à Christophe Colomb », p. 73-98) revient sur l'évolution de son image en Occident. Il montre que la figure du Khan se confond bien vite avec un mythe plus ancien et fortement ancré, celui du prêtre Jean. Alors que l'Occident découvre que le légendaire royaume chrétien n'existe 
pas, Marco Polo et les autres voyageurs inventent l'idée que les Mongols ont soumis le royaume chrétien, mais que leur chef était potentiellement un nouveau prêtre Jean. De là les sources chrétiennes font subir de nombreuses modifications idéologiques à cette figure historiographique connue par ailleurs, afin qu'elle corresponde à leurs attentes.

L'étude de M. Bais (p. 99-136) souligne la lecture idéologique des invasions Timourides chez T'ovma Mecop'ec'i (1368-1446), homme d'Église arménien qui obtint le titre de Vardapet, formé au monastère de Mecop', qu'il dirigea par la suite pendant une trentaine d'années. Auteur prolixe, il écrivit notamment une Histoire de Tamerlan et de ses successeurs, allant de 1386 aux années 1440 . Il s'agit de la principale source d'information sur ce qui se produisit en Arménie à cette époque. Témoin direct de l'invasion timouride puis des guerres entre chefs turcs, il utilise son récit pour montrer les tensions qui parcourent l'Église arménienne, notamment entre pro et anti-Latins. Lui-même participa activement au retour du siège du Catholicos de Sis, capitale d'Arménie cilicienne tombée en 1375, à Ejmiacin, en Grande Arménie, afin de limiter l'influence catholique sur l'église arménienne (ce qu'il obtint en 1441). Son histoire souligne la nécessaire unité de l'Église arménienne face à l'extérieur: les musulmans y sont présentés comme les bras armés de la punition divine, punition causée par l'hétérodoxie, source des luttes fratricides qui reproduisent le péché de Caïn (p. 122-123).

Un deuxième chapitre groupe des articles s'intéressant à la manière dont l'histoire fut utilisée dans le cadre de stratégies ecclésiologiques, afin de promouvoir l'unité et d'universalité de l'Église entre Arméniens, Grecs et Occidentaux. Dan Ioan Muresan (p. 139-206) dresse un bilan historiographique détaillé des discussions autour de la Donation de Constantin et du Constitutum Constantini. Il y discute les différentes hypothèses sur son auteur, sa date et son lieu de production : lui-même plaide pour une origine romaine et non franque (p. 183). Puis il livre une nouvelle lecture de la donation de Constantin : plutôt qu'un faux, il propose d'y voir un récit ecclésiologique, proche d'une compilation hagiographique intimement liée aux Actus Silvestri, même si par la suite, la narration d'origine est « reprise [...] sous les habits d'une constitution impériale », lui conférant une dimension juridique (p. 205). En appliquant les différentes étapes de la mimésis définies par Paul Ricœur, il montre la multiplicité des intentions et usages, variant entre temps d'écriture et temps de lecture. À travers une analyse linéaire du document, il discute de la nature du lien entre Église et Empire, puis montre comment ce texte a servi à affirmer l'aspect impérial et universel de la Papauté face aux églises d'Orient.

Yury Avvakumov (p. 207-236) étudie l'Anticimenon d'Anselme d'Havelberg (v. 1095-1158). Si le premier livre de cette œuvre aussi connue sous le titre de Dialogues est un traité sur l'unité de l'Église malgré les différentes formes de vie religieuse, les deux livres suivants contiennent un dialogue entre l'auteur et l'archevêque Nicétas de Nicomédie qui aurait eu lieu lors d'une ambassade à Constantinople en 1136. Les deux hommes auraient débattu des relations et différences entre l'Église romaine et grecque. L'état de la recherche sur l'œuvre et son auteur montre que pendant longtemps, les historiens ont utilisé le texte comme une source majeure sur les relations entre les deux églises, ne mettant pas en doute la réalité du dialogue. Soulignant les emprunts constants du texte à d'autres sources, mais aussi le fait que l'archevêque de Nicomédie en 1136 ne s'appelait pas Nicétas et qu'il avait des positions théologiques bien différentes du personnage du texte, l'auteur de l'étude considère que les détails "réalistes" du texte sont des trompel'œil. Il s'agit plutôt d'une œuvre de fiction, qui utilise une argumentation scolastique interne à l'Église d'Occident. Revenant sur la question de la place de l'eau dans l'eucharistie, Y. Avvakumov montre que l'œcuménisme d'Anselme est réel, mais qu'il ne cherche pas à reproduire fidèlement les positions $\mathrm{du}$ clergé byzantin : il veut plutôt évoquer la réponse de l'Église latine à certains débats théologiques.

Isabelle Augé (p. 237-249) s'intéresse à la confrontation entre Église arménienne et Église grecque dans les années 1165-1178, en se focalisant sur la Cilicie, zone d'échanges interculturels. Elle analyse et compare trois lettres du catholicos Grigor IV, qui œuvra beaucoup pour l'union des églises. On voit ainsi que l'on modifie et adapte la présentation des événements historiques en fonction des destinataires. Pour l'empereur grec Manuel Comnène, on souligne l'orthodoxie de l'Église arménienne. Écrivant aux docteurs de Grande Arménie, on insiste sur l'orthodoxie des Grecs. Pour cela, Grigor IV utilise les autorités conciliaires et patristiques en commun. 
Un dernier chapitre rassemble deux articles consacrés à la dimension eschatologique de l'histoire et à sa dimension prophétique. Felicitas Schmieder (p. 253-265) étudie 1'Expositio in Apocalipsim (v. 1248-49) d'Alexandre le Minorite, un Allemand du Nord proche des franciscains mais se disant laicus, qui écrit dans la veine de Joachim de Flore. Il lit l'Apocalypse de Jean au sens littéral, comme l'histoire du christianisme de Jésus à la fin des temps, et essaie d'identifier plusieurs figures et éléments avec ce qu'il connaît de l'histoire de la chrétienté. Cela produit un texte à mi-chemin entre le commentaire biblique et l'historiographie, se servant pour l'Occident de Frutolf de Michelsberg (et d'autres), et pour l'Orient de l'Historia Orientalis de Jacques de Vitry. L'Orient joue un rôle particulier, symbolique, dans son œuvre, et son histoire découvre ainsi nombre de figures prophétiques annonçant la fin des temps : Khosro II, Saladin ou Mahomet sont identifiés au dragon et à la bête, sans que le dernier soit toutefois explicitement identifié à l'Antéchrist.

S. La Porta (p. 267-303) s'intéresse à l'usage des prophéties autour de l'éclipse solaire de 1033, mille ans après la crucifixion de Jésus, qui fut interprétée en son temps comme la naissance de l'Antéchrist. Il montre que deux historiens postérieurs, Aristakes Lastivertc'i (qui écrit à la fin du $\mathrm{XI}^{\mathrm{e}} \mathrm{s}$.) et Matt'eos Urhayec'i (Mathieu d'Édesse), qui mène son récit jusqu'en 1128-1129, se sont servi des clés de lecture sorties de l'imaginaire prophétique pour pouvoir analyser l'histoire récente, pleine de bouleversements (domination byzantine suivie de l'irruption triomphale des Trucs à partir de 1071). En revanche, tous deux n'ont guère accordé de crédit à la dimension eschatologique de l'événement : ils furent conscients que si beaucoup en Arménie avaient vu dans l'éclipse un signe de l'Antéchrist et de la fin des temps, celle-ci ne s'est pas matérialisée. Le premier auteur place l'accomplissement de la prophétie au niveau historique et non eschatologique, sans attaquer directement les autorités éminentes qui avaient annoncé la naissance de l'Antéchrist. Mathieu d'Édesse cherche lui un nouveau sens eschatologique à la lumière des événements qui ont montré que la fin des temps n'était pas pour tout de suite : il voit dans les attaques des Seldjoukides une punition divine.

Les études de ce recueil sont fort intéressantes pour qui s'intéresse à l'histoire ecclésiastique ou l'histoire arménienne. L'accent a été mis sur les transferts de connaissances via les conciles, les réseaux monastiques et les contacts œcuméniques : c'est la force de l'ouvrage, qui livre une étude cohérente autour de la figure des Papes, des Patriarches et de l'histoire de l'Église, avec des études toutes éclairantes et solides. Mais c'est aussi sa faiblesse : on regrettera l'absence presque totale de la question du transfert de connaissances de l'historiographie musulmane, conséquence de l'approche ecclésiologique choisie par l'ouvrage. Comment l'histoire de l'Islam a-t-elle été transmise à l'Occident? Quel est le rôle des auteurs grecs et arméniens dans ce transfert? On évoque brièvement les nombreux détails que Jacques de Vitry donne sur la vie de Mahomet (p. 260) sans discuter de ses sources. L'histoire des souverains mongols est bien évoquée, mais les califes, les peuples musulmans sont très peu présents. Les rares articles qui évoquent la question (M. Bais et S. La Porta) abandonnent d'ailleurs la question de la transmission à l'Occident du savoir historique oriental : ils abordent le sujet d'un point de vue interne à l'historiographie arménienne.

Pierre Courroux 